\title{
Solutions for parametric double phase Robin problems
}

\author{
Nikolaos S. Papageorgiou ${ }^{\text {a }}$, Calogero Vetro ${ }^{\mathrm{b}, *}$ and Francesca Vetro ${ }^{\mathrm{c}, \mathrm{d}}$ \\ ${ }^{a}$ Department of Mathematics, Zografou campus, National Technical University, 15780, Athens, Greece \\ E-mail:npapg@math.ntua.gr \\ ${ }^{\mathrm{b}}$ Department of Mathematics and Computer Science, University of Palermo, Via Archirafi 34, 90123, \\ Palermo, Italy \\ E-mail: calogero.vetro@unipa.it \\ ${ }^{\mathrm{c}}$ Nonlinear Analysis Research Group, Ton Duc Thang University, Ho Chi Minh City, Vietnam \\ ${ }^{\mathrm{d}}$ Faculty of Mathematics and Statistics, Ton Duc Thang University, Ho Chi Minh City, Vietnam \\ E-mail:francescavetro@tdtu.edu.vn
}

Abstract. We consider a parametric double phase problem with Robin boundary condition. We prove two existence theorems. In the first the reaction is $(p-1)$-superlinear and the solutions produced are asymptotically big as $\lambda \rightarrow 0^{+}$. In the second the conditions on the reaction are essentially local at zero and the solutions produced are asymptotically small as $\lambda \rightarrow 0^{+}$.

Keywords: Unbalanced growth, asymptotically big solutions, asymptotically small solutions, superlinear reaction, $C$-condition

\section{Introduction}

Let $\Omega \subseteq \mathbb{R}^{N}$ be a bounded domain with a Lipschitz boundary $\partial \Omega$. In this paper we study the following parametric two phase Robin problem

$$
\left\{\begin{array}{l}
-\operatorname{div}\left(a(z)|\nabla u|^{p-2} \nabla u\right)-\Delta_{q} u+\xi(z)|u|^{p-2} u=\lambda f(z, u(z)) \quad \text { in } \Omega, \\
\frac{\partial u}{\partial n_{\vartheta}}+\beta(z)|u|^{p-2} u=0 \quad \text { on } \partial \Omega, 1<q<p<+\infty
\end{array}\right.
$$

In this problem $a \in L^{\infty}(\Omega)$ with $a(z)>0$ for a.a. $z \in \Omega$ and $\Delta_{q}$ denotes the $q$-Laplace differential operator defined by

$$
\Delta_{q} u=\operatorname{div}\left(|\nabla u|^{q-2} \nabla u\right) \quad \text { for all } W^{1, q}(\Omega) .
$$

The differential operator in problem $\left(P_{\lambda}\right)$ is related to the two-phase integral functional

$$
u \rightarrow \int_{\Omega}\left[a(z)|\nabla u|^{p}+|\nabla u|^{q}\right] d z .
$$

\footnotetext{
*Corresponding author. E-mail: calogero.vetro@unipa.it.
} 
In the integral functional, the integrand is the function

$$
\vartheta(z, y)=a(z)|y|^{p}+|y|^{q} \quad \text { for all } z \in \Omega \text {, all } y \in \mathbb{R}^{N} .
$$

Since we do not assume that the coefficient $a(\cdot)$ is bounded away from zero, this integrand exhibits unbalanced growth, namely we have

$$
|y|^{q} \leqslant \vartheta(z, y) \leqslant c_{0}\left[1+|y|^{p}\right] \text { for some } c_{0}>0, \text { all } z \in \Omega \text {, all } y \in \mathbb{R}^{N} .
$$

Such functionals were investigated first in the context of problems related to elasticity theory, by Marcellini [10] and Zhikov [20]. Recently the interest for such functional was revived with the remarkable works of Mingione and coworkers (see Baroni-Colombo-Mingione [1], Colombo-Mingione [3,4], De Filippis-Mingione [5]), who proved local regularity results for minimizers of such functionals. A global regularity theory is still elusive and so the tools and techniques used in the study of $(p, q)$-equations (see, for example, Papageorgiou-Vetro-Vetro [15]) are not applicable in two-phase problems. Even the ambient space changes and it is no longer the Sobolev space $W^{1, p}(\Omega)$, but the Musielak-OrliczSobolev space $W^{1, \vartheta}(\Omega)$ (see Section 2). In the left hand side of $\left(P_{\lambda}\right)$ we also have a potential term $x \rightarrow \xi(z)|x|^{p-2} x$ with $\xi \in L^{\infty}(\Omega), \xi(z) \geqslant 0$ for a.a. $z \in \Omega$. The reaction $\lambda f(z, x)$ is parametric, with $\lambda>0$ being the parameter and $f(z, x)$ is a Carathéodory function (that is, for all $x \in \mathbb{R}, z \rightarrow f(z, x)$ is measurable and for a.a. $z \in \Omega, x \rightarrow f(z, x)$ is continuous). We prove two existence theorems and provide information about the asymptotic behavior of the solutions as $\lambda \rightarrow 0^{+}$. In the first existence theorem we assume that $f(z, \cdot)$ exhibits $(p-1)$-superlinear growth near $\pm \infty$. However, we do not employ the Ambrosetti-Rabinowitz condition (the AR-condition for short), which is common in the literature when dealing with superlinear problems. In this case we show that for the solution $u_{\lambda}$, we have $\left\|u_{\lambda}\right\| \rightarrow+\infty$ as $\lambda \rightarrow 0^{+}$. In the second, the hypotheses on $f(z, \cdot)$, aside from the "subcritical" growth condition, concern only its behavior near zero. In this case we show that $\left\|u_{\lambda}\right\| \rightarrow 0^{+}$as $\lambda \rightarrow 0^{+}$. In the boundary condition $\frac{\partial u}{\partial n_{\vartheta}}$ denotes the conormal derivative of $u$ with respect to the modular function $\vartheta$. We interpret this derivative using the nonlinear Green's identity (see Papageorgiou-Rădulescu-Repovš [11], Corollary 1.5.16, p. 34). When $u \in C^{1}(\bar{\Omega})$, we have

$$
\frac{\partial u}{\partial n_{\vartheta}}=\left[a(z)|\nabla u|^{p-2}+|\nabla u|^{q-2}\right] \frac{\partial u}{\partial n},
$$

with $n(\cdot)$ being the outward unit normal on $\partial \Omega$.

We mention that recently existence and multiplicity results for two phase problems were proved by Gasiński-Papageorgiou [6], Ge-Lv-Lu [7], Liu-Dai [9], Papageorgiou-Rădulescu-Repovš [12-14], Papageorgiou-Vetro-Vetro [16]. In the framework of double-phase problems with variable growth we refer to Cencelj-Rădulescu-Repovš [2], Ragusa-Tachikawa [18] and Zhang-Rădulescu [19].

\section{Mathematical background - Hypotheses}

As we already mentioned in the Introduction, the right function space framework for the analysis of problem $\left(P_{\lambda}\right)$ is provided by the so-called Musielak-Orlicz-Sobolev spaces.

We consider the Carathéodory function

$$
\vartheta(z, x)=a(z) x^{p}+x^{q} \quad \text { for all } z \in \Omega \text {, all } x \geqslant 0 .
$$


Then the Musielak-Orlicz space $L^{\vartheta}(\Omega)$ is defined by

$$
L^{\vartheta}(\Omega)=\left\{u: \Omega \rightarrow \mathbb{R} \text { is measurable and } \rho_{\vartheta}(u)=\int_{\Omega} \vartheta(z,|u|) d z<+\infty\right\} .
$$

We furnish $L^{\vartheta}(\Omega)$ with the so-called "Luxemburg norm" defined by

$$
\|u\|_{\vartheta}=\inf \left[\lambda>0: \rho_{\vartheta}\left(\frac{u}{\lambda}\right) \leqslant 1\right]
$$

Then $L^{\vartheta}(\Omega)$ becomes a separable, reflexive (in fact uniformly convex) Banach space. Also, we introduce the weighted Lebesgue space

$$
L_{a}^{p}(\Omega)=\left\{u: \Omega \rightarrow \mathbb{R} \text { is measurable and }\|u\|_{a, p}=\left[\int_{\Omega} a(z)|u|^{p} d z\right]^{1 / p}<+\infty\right\} .
$$

We know that

$$
L^{p}(\Omega) \hookrightarrow L^{\vartheta}(\Omega) \hookrightarrow L^{q}(\Omega) \cap L_{a}^{p}(\Omega),
$$

and $\min \left\{\|u\|_{\vartheta}^{p},\|u\|_{\vartheta}^{q}\right\} \leqslant\|u\|_{q}^{q}+\|u\|_{a, p}^{p} \leqslant \max \left\{\|u\|_{\vartheta}^{p},\|u\|_{\vartheta}^{q}\right\}$ for all $u \in L^{\vartheta}(\Omega)$.

Then, we can define the corresponding Sobolev-type space $W^{1, \vartheta}(\Omega)$ by setting

$$
W^{1, \vartheta}(\Omega)=\left\{u \in L^{\vartheta}(\Omega):|\nabla u| \in L^{\vartheta}(\Omega)\right\} .
$$

We furnish $W^{1, \vartheta}(\Omega)$ with the norm

$$
\|u\|=\|u\|_{\vartheta}+\|\nabla u\|_{\vartheta} \quad \text { for all } u \in W^{1, \vartheta}(\Omega)
$$

(here $\|\nabla u\|_{\vartheta}=\||\nabla u|\|_{\vartheta}$ ). Normed this way, the space $W^{1, \vartheta}(\Omega)$ is separable and reflexive (in fact uniformly convex). We know that

$$
W^{1, \vartheta}(\Omega) \hookrightarrow L^{r}(\Omega) \text { compactly }
$$

for every $r \in\left(1, q^{*}\right)$ with

$$
q^{*}= \begin{cases}\frac{N q}{N-q} & \text { if } q<N, \\ +\infty & \text { if } N \leqslant q\end{cases}
$$

(the critical Sobolev exponent corresponding to $q$ ).

On $\partial \Omega$ we consider the $(N-1)$-dimensional Hausdorff measure (surface measure) $\sigma(\cdot)$. Using this measure, we can define in the usual way the boundary Lebesgue spaces $L^{s}(\partial \Omega)(1 \leqslant s \leqslant+\infty)$. We know that there exists a unique continuous linear map $\gamma_{0}: W^{1, q}(\Omega) \rightarrow L^{q}(\partial \Omega)$, known as the "trace map", such that

$$
\gamma_{0}(u)=\left.u\right|_{\partial \Omega} \quad \text { for all } u \in W^{1, q}(\Omega) \cap C(\bar{\Omega}) .
$$


The trace map extends the notion of boundary values to all Sobolev functions. We know that

$$
\operatorname{im} \gamma_{0}=W^{\frac{1}{q^{\prime}}, q}(\partial \Omega) \quad\left(\frac{1}{q}+\frac{1}{q^{\prime}}=1\right) \quad \text { and } \quad \operatorname{ker} \gamma_{0}=W_{0}^{1, q}(\Omega)
$$

Moreover, the trace map is compact into $L^{s}(\partial \Omega)$ for all $s \in\left[1, \frac{(N-1) q}{N-q}\right)$ if $q<N$ and into $L^{s}(\partial \Omega)$ for all $s \geqslant 1$ if $q \geqslant N$. In the sequel, for the sake of notational simplicity, we drop the use of the trace map $\gamma_{0}(\cdot)$. All restrictions of Sobolev functions on $\partial \Omega$ are understood in the sense of traces.

If $X$ is a Banach space and $\varphi \in C^{1}(X, \mathbb{R})$, then we say that $\varphi(\cdot)$ satisfies the " $C$-condition", if every sequence $\left\{u_{n}\right\}_{n} \geqslant 1 \subseteq X$ such that $\left\{\varphi\left(u_{n}\right)\right\}_{n} \geqslant 1 \subseteq \mathbb{R}$ is bounded and $\left(1+\left\|u_{n}\right\|_{X}\right) \varphi^{\prime}\left(u_{n}\right) \rightarrow 0$ in $X^{*}$ as $n \rightarrow+\infty$, admits a strongly convergent subsequence. Also by $K_{\varphi}$ we denote the critical set of $\varphi$, that is, $K_{\varphi}=\left\{u \in X: \varphi^{\prime}(u)=0\right\}$.

Let $A: W^{1, \vartheta}(\Omega) \rightarrow W^{1, \vartheta}(\Omega)^{*}$ be the nonlinear map defined by

$$
\langle A(u), h\rangle=\int_{\Omega}\left[a(z)|\nabla u|^{p-2}+|\nabla u|^{q-2}\right](\nabla u, \nabla h)_{\mathbb{R}^{N}} d z \quad \text { for all } u, h \in W^{1, \vartheta}(\Omega) .
$$

This map has the following properties (see Liu-Dai [9], Proposition 3.1).

Proposition 1. If $a \in L^{\infty}(\Omega)$ and $a(z)>0$ for a.a. $z \in \Omega$, then $A(\cdot)$ is bounded (that is, maps bounded sets to bounded sets), continuous, monotone (hence maximal monotone too) and of type $(S)_{+}$(that is, if $u_{n} \stackrel{w}{\rightarrow} u$ in $W^{1, \vartheta}(\Omega)$ and $\lim \sup _{n \rightarrow+\infty}\left\langle A\left(u_{n}\right), u_{n}-u\right\rangle \leqslant 0$, then $u_{n} \rightarrow u$ in $\left.W^{1, \vartheta}(\Omega)\right)$.

The hypotheses on the data of $\left(P_{\lambda}\right)$ are the following:

$H_{0}: a \in L^{\infty}(\Omega)$ with $a(z) \geqslant 0$ for a.a. $z \in \Omega, \xi \in L^{\infty}(\Omega)$ with $\xi(z) \geqslant 0$ for a.a. $z \in \Omega, \beta \in L^{\infty}(\partial \Omega)$ with $\beta(z) \geqslant 0$ for $\sigma$-a.a. $z \in \partial \Omega, \xi \not \equiv 0$ or $\beta \not \equiv 0$ and $\frac{N p}{N+p-1}<q$.

Remark 1. The last condition in hypotheses $H_{0}$, which relates the two exponents $p$ and $q$, implies that $W^{1, \vartheta}(\Omega) \hookrightarrow L^{p}(\partial \Omega)$ compactly via the trace map $\gamma_{0}(\cdot)$.

$H_{1}: f: \Omega \times \mathbb{R} \rightarrow \mathbb{R}$ is a Carathéodory function such that $f(z, 0)=0$ for a.a. $z \in \Omega$ and

(i) $|f(z, x)| \leqslant \widehat{a}(z)\left[1+|x|^{r-1}\right]$ for a.a. $z \in \Omega$, all $x \in \mathbb{R}$, with $\widehat{a} \in L^{\infty}(\Omega), p<r<q^{*}$;

(ii) if $F(z, x)=\int_{0}^{x} f(z, s) d s$, then $\lim _{x \rightarrow \pm \infty} \frac{F(z, x)}{|x|^{p}}=+\infty$ uniformly for a.a. $z \in \Omega$;

(iii) there exists $\tau \in\left((r-q) \max \left\{1, \frac{N}{q}\right\}, q^{*}\right)$ with $\tau>q$ such that

$$
0<\widehat{\eta} \leqslant \liminf _{x \rightarrow \pm \infty} \frac{f(z, x) x-p F(z, x)}{|x|^{\tau}} \quad \text { uniformly for a.a. } z \in \Omega
$$

(iv) there exist $1<\mu<q$ and $c_{1}>0$ such that

$$
-c_{1} \leqslant \liminf _{x \rightarrow 0} \frac{F(z, x)}{|x|^{\mu}} \leqslant \limsup _{x \rightarrow 0} \frac{F(z, x)}{|x|^{\mu}} \leqslant c_{1} \quad \text { uniformly for a.a. } z \in \Omega .
$$


Remark 2. From hypotheses $H_{1}$ (ii), (iii), we have that

$$
\lim _{x \rightarrow \pm \infty} \frac{f(z, x)}{|x|^{p-2} x}=+\infty \quad \text { uniformly for a.a. } z \in \Omega
$$

So the reaction $f(z, \cdot)$ is $(p-1)$-superlinear. However, this superlinear growth of $f(z, \cdot)$ is not expressed using the AR-condition. Recall that the AR-condition says that there exist $\eta>p$ and $M>0$ such that

$$
\begin{aligned}
& 0<\eta F(z, x) \leqslant f(z, x) x \quad \text { for a.a. } z \in \Omega, \text { all }|x| \geqslant M, \\
& 0<\underset{\Omega}{\operatorname{essinf}} F(\cdot, \pm M) .
\end{aligned}
$$

Integrating (1a) and using (1b), we obtain the following weaker condition

$$
\begin{gathered}
c_{2}|x|^{\eta} \leqslant F(z, x) \quad \text { for a.a. } z \in \Omega \text {, all }|x| \geqslant M, \text { some } c_{2}>0 \\
\Rightarrow \quad c_{2}|x|^{\eta} \leqslant f(z, x) x \quad \text { for a.a. } z \in \Omega, \text { all }|x| \geqslant M .
\end{gathered}
$$

In this paper instead of the AR-condition, we employ hypothesis $H_{1}$ (iii) which is less restrictive and incorporates in our framework superlinear nonlinearities which fail to satisfy the AR-condition. For example consider the following function (for the sake of simplicity we drop the $z$-dependence)

$$
f(x)= \begin{cases}|x|^{\mu-2} x & \text { if }|x| \leqslant 1, \\ |x|^{p-2} x \ln |x|+|x|^{s-2} x & \text { if } 1<|x|,\end{cases}
$$

with $1<\mu<q$ and $1<s<p$. The function satisfies hypothesis $H_{1}$, but fails to satisfy the ARcondition.

Let $\widehat{\gamma}_{p}: W^{1, \vartheta}(\Omega) \rightarrow \mathbb{R}$ be the $C^{1}$-functional defined by

$$
\widehat{\gamma}_{p}(u)=\int_{\Omega} a(z)|\nabla u|^{p} d z+\int_{\Omega} \xi(z)|u|^{p} d z+\int_{\partial \Omega} \beta(z)|u|^{p} d \sigma \quad \text { for all } u \in W^{1, \vartheta}(\Omega) .
$$

Proposition 2. If hypotheses $H_{0}$ hold, then $c_{3}\|u\|^{p} \leqslant \widehat{\gamma}_{p}(u)$ for some $c_{3}>0$, all $u \in W^{1, \vartheta}(\Omega)$.

Proof. We argue by contradiction. So, suppose that the result of the proposition is not true. Then on account of the $p$-homogeneity of $\widehat{\gamma}_{p}(\cdot)$, we can find $\left\{u_{n}\right\}_{n} \geqslant 1 \subseteq W^{1, \vartheta}(\Omega)$ such that

$$
\left\|u_{n}\right\|=1 \quad \text { and } \quad \widehat{\gamma}_{p}\left(u_{n}\right)<\frac{1}{n} \quad \text { for all } n \in \mathbb{N} .
$$

We may assume that

$$
u_{n} \stackrel{w}{\rightarrow} u \quad \text { in } W^{1, \vartheta}(\Omega) \quad \text { and } \quad u_{n} \rightarrow u \quad \text { in } L^{p}(\Omega) \text { and in } L^{p}(\partial \Omega) .
$$


From (2) and (3) it follows that

$$
\begin{aligned}
& \int_{\Omega} a(z)|\nabla u|^{p} d z=0 \\
& \quad \Rightarrow \quad|\nabla u(z)|=0 \quad \text { for a.a. } z \in \Omega \\
& \Rightarrow \quad u \equiv c \in \mathbb{R} .
\end{aligned}
$$

Then from (2) in the limit as $n \rightarrow+\infty$ we have

$$
\begin{gathered}
|c|^{p}\left[\int_{\Omega} \xi(z) d z+\int_{\partial \Omega} \beta(z) d \sigma\right]=0 \\
\Rightarrow \quad c=0 \quad\left(\text { see hypotheses } H_{0}\right) \\
\Rightarrow \quad u_{n} \rightarrow 0 \quad \text { in } W^{1, \vartheta}(\Omega),
\end{gathered}
$$

which contradicts (2).

For every $\lambda>0$, let $\varphi_{\lambda}: W^{1, \vartheta}(\Omega) \rightarrow \mathbb{R}$ be the energy (Euler) functional for problem $\left(P_{\lambda}\right)$ defined by

$$
\varphi_{\lambda}(u)=\frac{1}{p} \widehat{\gamma}_{p}(u)+\frac{1}{q}\|\nabla u\|_{q}^{q}-\lambda \int_{\Omega} F(z, u) d z \quad \text { for all } u \in W^{1, p}(\Omega) .
$$

Evidently $\varphi_{\lambda} \in C^{1}\left(W^{1, \vartheta}(\Omega), \mathbb{R}\right)$.

\section{Asymptotically big solutions}

In this section we show that for all $\lambda>0$ small problem $\left(P_{\lambda}\right)$ has a solution $u_{\lambda} \in W^{1, \vartheta}(\Omega)$ such that $\left\|u_{\lambda}\right\| \rightarrow+\infty$ as $\lambda \rightarrow 0^{+}$.

Proposition 3. If hypotheses $H_{0}, H_{1}$ hold and $\lambda>0$, then the functional $\varphi_{\lambda}(\cdot)$ satisfies the $C$-condition.

Proof. We consider a sequence $\left\{u_{n}\right\}_{n} \geqslant 1 \subseteq W^{1, \vartheta}(\Omega)$ such that

$$
\begin{aligned}
& \left|\varphi_{\lambda}\left(u_{n}\right)\right| \leqslant c_{4} \text { for some } c_{4}>0, \text { all } n \in \mathbb{N}, \\
& \left(1+\left\|u_{n}\right\|\right) \varphi_{\lambda}^{\prime}\left(u_{n}\right) \rightarrow 0 \quad \text { in } W^{1, \vartheta}(\Omega)^{*} \text { as } n \rightarrow+\infty .
\end{aligned}
$$

From (5) we have

$$
\begin{aligned}
& \left.\left|\left\langle A\left(u_{n}\right), h\right\rangle+\int_{\Omega} \xi(z)\right| u_{n}\right|^{p-2} u_{n} h d z+\int_{\partial \Omega} \beta(z)\left|u_{n}\right|^{p-2} u_{n} h d \sigma-\lambda \int_{\Omega} f\left(z, u_{n}\right) h d z \mid \\
& \leqslant \frac{\varepsilon_{n}\|h\|}{1+\left\|u_{n}\right\|} \quad \text { for all } h \in W^{1, \vartheta}(\Omega), \text { with } \varepsilon_{n} \rightarrow 0^{+} .
\end{aligned}
$$


In (6) we choose $h=u_{n} \in W^{1, \vartheta}(\Omega)$ and obtain

$$
-\widehat{\gamma}_{p}\left(u_{n}\right)-\left\|\nabla u_{n}\right\|_{q}^{q}+\lambda \int_{\Omega} f\left(z, u_{n}\right) u_{n} d z \leqslant \varepsilon_{n} \quad \text { for all } n \in \mathbb{N} .
$$

Also from (4) we have

$$
\widehat{\gamma}_{p}\left(u_{n}\right)+\frac{p}{q}\left\|\nabla u_{n}\right\|_{q}^{q}-\lambda \int_{\Omega} p F\left(z, u_{n}\right) d z \leqslant p c_{4} \quad \text { for all } n \in \mathbb{N} .
$$

We add (7) and (8) and recall that $q<p$. Then

$$
\lambda \int_{\Omega}\left[f\left(z, u_{n}\right) u_{n}-p F\left(z, u_{n}\right)\right] d z \leqslant c_{5} \quad \text { for some } c_{5}>0, \text { all } n \in \mathbb{N} .
$$

Hypotheses $H_{1}(\mathrm{i})$, (iii) imply that

$$
c_{6}|x|^{\tau}-c_{7} \leqslant f(z, x) x-p F(z, x) \text { for a.a. } z \in \Omega \text {, all } x \in \mathbb{R} \text {, some } c_{6}, c_{7}>0 .
$$

We use (10) in (9) and obtain

$$
\begin{array}{r}
\left\|u_{n}\right\|_{\tau}^{\tau} \leqslant c_{8} \quad \text { for some } c_{8}>0, \text { all } n \in \mathbb{N} \\
\Rightarrow \quad\left\{u_{n}\right\}_{n} \geqslant 1 \subseteq L^{\tau}(\Omega) \quad \text { is bounded. }
\end{array}
$$

First assume that $q<N$. From hypothesis $H_{1}$ (iii) it is clear that we may assume that $\tau<r<q^{*}$. Let $t \in(0,1)$ be such that

$$
\frac{1}{r}=\frac{1-t}{\tau}+\frac{t}{q^{*}}
$$

Using the interpolation inequality (see Papageorgiou-Winkert [17], Proposition 2.3.17, p. 116), we have

$$
\begin{aligned}
&\left\|u_{n}\right\|_{r} \leqslant\left\|u_{n}\right\|_{\tau}^{1-t}\left\|u_{n}\right\|_{q^{*}}^{t} \\
& \Rightarrow \quad\left\|u_{n}\right\|_{r}^{r} \leqslant c_{9}\left\|u_{n}\right\|^{t r} \quad \text { for some } c_{9}>0, \text { all } n \in \mathbb{N} \\
&\left(\text { see }(11) \text { and recall that } W^{1, \vartheta}(\Omega) \hookrightarrow L^{q^{*}}(\Omega)\right) .
\end{aligned}
$$

From (6) with $h=u_{n} \in W^{1, \vartheta}(\Omega)$ we obtain

$$
\begin{aligned}
& \widehat{\gamma}_{p}\left(u_{n}\right)+\left\|\nabla u_{n}\right\|_{q}^{q}-\lambda \int_{\Omega} f\left(z, u_{n}\right) u_{n} d z \leqslant \varepsilon_{n} \quad \text { for all } n \in \mathbb{N} \\
& \Rightarrow \quad c_{3}\left\|u_{n}\right\|^{p} \leqslant \lambda \int_{\Omega} f\left(z, u_{n}\right) u_{n} d z+\varepsilon_{n} \quad \text { (see Proposition 2) } \\
& \leqslant \lambda c_{10}\left[1+\left\|u_{n}\right\|^{t r}\right]+\varepsilon_{n} \quad \text { for some } c_{10}>0, \text { all } n \in \mathbb{N} \\
&\left(\text { see hypothesis } H_{1}(\mathrm{i}) \text { and }(13)\right) .
\end{aligned}
$$


From (12) we have

$$
\begin{aligned}
t & =\frac{q^{*}(r-\tau)}{r\left(q^{*}-\tau\right)} \\
& \Rightarrow \quad t r=\frac{q^{*}(r-\tau)}{q^{*}-\tau} .
\end{aligned}
$$

On account of hypothesis $H_{1}$ (iii) we have

$$
\begin{aligned}
& (r-q) \frac{N}{q}<\tau \quad(\text { recall that we have assumed that } q<N) \\
& \Rightarrow \quad N(r-q)<\tau q \\
& \Rightarrow \quad N r-N \tau<N q-N \tau+\tau q \\
& \Rightarrow \quad \frac{N q(r-\tau)}{N q-N \tau+\tau q}<q \\
& \Rightarrow \quad \frac{q^{*}(r-\tau)}{q^{*}-\tau}<q \\
& \Rightarrow \quad \operatorname{tr}<q \quad(\text { see }(15))
\end{aligned}
$$

Then from (14) and since $q<p$, we infer that

$$
\left\{u_{n}\right\}_{n \geqslant 1} \subseteq W^{1, \vartheta}(\Omega) \quad \text { is bounded. }
$$

Next suppose that $q \geqslant N$. In this case we know that $q^{*}=+\infty$, while from the Sobolev embedding theorem, we have

$$
W^{1, \vartheta}(\Omega) \hookrightarrow W^{1, q}(\Omega) \hookrightarrow L^{s}(\Omega) \quad \text { (for all } 1 \leqslant s<+\infty \text { ). }
$$

So, in the previous argument we need to replace $q^{*}$ by $l>r$.

Then again from (12) we have

$$
\left.\operatorname{tr}=\frac{l(r-\tau)}{l-\tau} \rightarrow r-\tau<q \quad \text { as } l \rightarrow+\infty \text { (see hypothesis } H_{1}(\mathrm{iii})\right) .
$$

So, by choosing $l>r$ big, we will have

$$
\operatorname{tr}<q<p
$$

hence (16) holds again.

From (16) it follows that we may assume that

$$
u_{n} \stackrel{w}{\rightarrow} u \quad \text { in } W^{1, \vartheta}(\Omega) \quad \text { and } \quad u_{n} \rightarrow u \quad \text { in } L^{p}(\Omega) \text { and in } L^{p}(\partial \Omega) .
$$


In (6) we choose $h=u_{n}-u \in W^{1, \vartheta}(\Omega)$, pass to the limit as $n \rightarrow+\infty$ and use (17). Then

$$
\begin{aligned}
& \lim _{n \rightarrow+\infty}\left\langle A\left(u_{n}\right), u_{n}-u\right\rangle=0 \\
& \quad \Rightarrow \quad u_{n} \rightarrow u \text { in } W^{1, \vartheta}(\Omega)(\text { see Proposition } 1) .
\end{aligned}
$$

We conclude that for every $\lambda>0$ the functional $\varphi_{\lambda}(\cdot)$ satisfies the $C$-condition.

Proposition 4. If hypotheses $H_{0}, H_{1}$ hold, then we can find $\lambda^{*}>0$ such that $0<m_{\lambda} \leqslant \varphi_{\lambda}(u)$ for all $\|u\|=\rho_{\lambda}$, all $\lambda \in\left(0, \lambda^{*}\right)$.

Proof. On account of hypotheses $H_{1}(\mathrm{i})$, (iv), we have

$$
|F(z, x)| \leqslant c_{11}\left[|x|^{\mu}+|x|^{r}\right] \quad \text { for a.a. } z \in \Omega \text {, all } x \in \mathbb{R} \text {, some } c_{11}>0 \text {. }
$$

Then for every $u \in W^{1, \vartheta}(\Omega)$ we have

$$
\varphi_{\lambda}(u) \geqslant \frac{c_{3}}{p}\|u\|^{p}-\lambda c_{12}\left[\|u\|^{\mu}+\|u\|^{r}\right] \quad \text { for some } c_{12}>0
$$

(see Proposition 2 and (18)).

Consider $u \in W^{1, \vartheta}(\Omega)$ with $\|u\|=\rho_{\lambda}=\lambda^{-\delta}$ where $0<\delta<\frac{1}{r-p}$. Then from (19) we have

$$
\begin{aligned}
\varphi_{\lambda}(u) & \geqslant \frac{c_{3}}{p} \lambda^{-\delta p}-c_{12}\left[\lambda^{1-\delta \mu}+\lambda^{1-\delta r}\right] \\
& =\left[\frac{c_{3}}{p}-c_{12}\left(\lambda^{1-\delta(\mu-p)}+\lambda^{1-\delta(r-p)}\right)\right] \lambda^{-\delta p}=m_{\lambda} .
\end{aligned}
$$

Note that

$$
0<1-\delta(r-p)<1-\delta(\mu-p)
$$

Then we can find $\lambda^{*}>0$ such that

$$
\lambda^{1-\delta(\mu-p)}+\lambda^{1-\delta(r-p)}<\frac{c_{3}}{c_{12} p} \text { for all } \lambda \in\left(0, \lambda^{*}\right) .
$$

From (20) we infer that

$$
\varphi_{\lambda}(u) \geqslant m_{\lambda}>0 \quad \text { for all } u \in W^{1, \vartheta}(\Omega) \text { with }\|u\|=\rho_{\lambda} \text {, all } 0<\lambda<\lambda^{*} .
$$

Remark 3. From the above proof we see that $m_{\lambda} \rightarrow+\infty$ as $\lambda \rightarrow 0^{+}$(see (20)).

Now we can produce solutions of $\left(P_{\lambda}\right)$ which asymptotically as $\lambda \rightarrow 0^{+}$become arbitrarily big in the $W^{1, \vartheta}(\Omega)$-norm. 
Theorem 1. If hypotheses $H_{0}, H_{1}$ hold, then we can find $\lambda^{*}>0$ such that for all $\lambda \in\left(0, \lambda^{*}\right)$ problem $\left(P_{\lambda}\right)$ has a nontrivial solution $u_{\lambda} \in W^{1, \vartheta}(\Omega)$ and $\left\|u_{\lambda}\right\| \rightarrow+\infty$ as $\lambda \rightarrow 0^{+}$.

Proof. Let $u \in W^{1, \vartheta}(\Omega)$ with $u(z)>0$ for a.a. $z \in \Omega$. Then on account of hypothesis $H_{1}(i i)$ we have

$$
\varphi_{\lambda}(t u) \rightarrow-\infty \quad \text { as } t \rightarrow+\infty
$$

Then (21) together with Propositions 3 and 4, permit the use of the mountain pass theorem. So, we can find $u_{\lambda} \in W^{1, \vartheta}(\Omega)$ such that

$$
u_{\lambda} \in K_{\varphi_{\lambda}} \quad \text { and } \quad \varphi_{\lambda}(0)=0<m_{\lambda} \leqslant \varphi_{\lambda}\left(u_{\lambda}\right) .
$$

So, $u_{\lambda}$ is a nontrivial solution of $\left(P_{\lambda}\right)\left(\lambda \in\left(0, \lambda^{*}\right)\right)$. Using (18), we have

$$
\begin{aligned}
& \varphi_{\lambda}\left(u_{\lambda}\right) \leqslant c_{13}\left[\left\|u_{\lambda}\right\|^{p}+\left\|u_{\lambda}\right\|^{\mu}+\left\|u_{\lambda}\right\|^{r}\right] \quad \text { for some } c_{13}>0 \\
& \left.\quad \Rightarrow \quad m_{\lambda} \leqslant c_{14}\left[1+\left\|u_{\lambda}\right\|^{r}\right] \quad \text { for some } c_{14}>0 \text { (see (22) and recall that } 1<\mu<p<r\right) \\
& \quad \Rightarrow \quad\left\|u_{\lambda}\right\| \rightarrow+\infty \quad \text { as } \lambda \rightarrow 0^{+} \text {(recall that } m_{\lambda} \rightarrow+\infty \text { as } \lambda \rightarrow 0^{+} \text {). }
\end{aligned}
$$

\section{Asymptotically small solutions}

In this section, we provide conditions on $f(z, x)$ which guarantee that for all $\lambda>0$ small problem $\left(P_{\lambda}\right)$ has a solution $\widehat{u}_{\lambda} \in W^{1, \vartheta}(\Omega)$ such that $\left\|\widehat{u}_{\lambda}\right\| \rightarrow 0^{+}$as $\lambda \rightarrow 0^{+}$.

The new conditions on the function $f(z, x)$ in the reaction are the following:

$H_{2}: f: \Omega \times \mathbb{R} \rightarrow \mathbb{R}$ is a Carathéodory function such that $f(z, 0)=0$ for a.a. $z \in \Omega$ and

(i) $|f(z, x)| \leqslant \widehat{a}(z)\left[1+|x|^{r-1}\right]$ for a.a. $z \in \Omega$, all $x \in \mathbb{R}$, with $\widehat{a} \in L^{\infty}(\Omega), p<r<q^{*}$;

(ii) there exists $\tau \in(1, q)$ and $\delta, \widehat{c}, \widetilde{c}$ such that

$$
\begin{aligned}
& \widehat{c}|x|^{\tau} \leqslant F(z, x) \quad \text { for a.a. } z \in \Omega, \text { all }|x| \leqslant \delta, \\
& \limsup _{x \rightarrow 0} \frac{F(z, x)}{|x|^{\tau}} \leqslant \widetilde{c} \quad \text { uniformly for a.a. } z \in \Omega .
\end{aligned}
$$

Remark 4. The hypotheses on $f(z, \cdot)$ are minimal. We stress that no asymptotic condition as $x \rightarrow \pm \infty$ is imposed on $f(z, \cdot)$. Only the subcritical growth condition $H_{2}(\mathrm{i})$, which guarantees that the energy functional of the problem is $\mathrm{C}^{1}$. It is an interesting open question whether we can drop hypothesis $\mathrm{H}_{2}(\mathrm{i})$ and use cut-off techniques like those in Leonardi-Papageorgiou [8]. The lack of global regularity results for double phase problems, make such an approach problematic.

Theorem 2. If hypotheses $H_{0}, H_{2}$ hold, then we can find $\widehat{\lambda}^{*}>0$ such that for all $\lambda \in\left(0, \widehat{\lambda}^{*}\right)$ problem $\left(P_{\lambda}\right)$ has a nontrivial solution $\widehat{u}_{\lambda} \in W^{1, \vartheta}(\Omega)$ and $\left\|\widehat{u}_{\lambda}\right\| \rightarrow 0^{+}$as $\lambda \rightarrow 0^{+}$.

Proof. As before $\varphi_{\lambda}: W^{1, \vartheta}(\Omega) \rightarrow \mathbb{R}$ is the energy functional for problem $\left(P_{\lambda}\right)$ defined by

$$
\varphi_{\lambda}(u)=\frac{1}{p} \widehat{\gamma}_{p}(u)+\frac{1}{q}\|\nabla u\|_{q}^{q}-\lambda \int_{\Omega} F(z, u) d z \quad \text { for all } u \in W^{1, \vartheta}(\Omega) .
$$


We know that $\varphi_{\lambda} \in C^{1}\left(W^{1, \vartheta}(\Omega), \mathbb{R}\right)$. Hypotheses $H_{2}$ imply that

$$
|F(z, x)| \leqslant c_{15}\left[|x|^{\tau}+|x|^{r}\right] \quad \text { for a.a. } z \in \Omega \text {, all } x \in \mathbb{R} \text {, some } c_{15}>0 \text {. }
$$

Let $0<\delta<\frac{1}{p}$. Then for $u \in W^{1, \vartheta}(\Omega)$ with $\|u\|=\lambda^{\delta}$, we have

$$
\begin{aligned}
\varphi_{\lambda}(u) & \geqslant \frac{c_{3}}{p} \lambda^{\delta p}-c_{16}\left[\lambda^{\delta \tau}+\lambda^{\delta r}\right] \quad \text { for some } c_{15}>0(\text { see Proposition } 1 \text { and (23)) } \\
& =\left[\frac{c_{3}}{p} \lambda^{\delta p-1}-c_{16}\left(\lambda^{\delta \tau}+\lambda^{\delta r}\right)\right] \lambda .
\end{aligned}
$$

Note that $\delta p-1<0$ and so we see that we can find $\widehat{\lambda}^{*}>0$ such that for all $\lambda \in\left(0, \widehat{\lambda}^{*}\right)$ we have

$$
\varphi_{\lambda}(u)>0 \quad \text { for all } u \in W^{1, \vartheta}(\Omega) \text { with }\|u\|=\lambda^{\delta} .
$$

Let $B_{\lambda}=\left\{u \in W^{1, \vartheta}(\Omega):\|u\|<\lambda^{\delta}\right\}$. The reflexivity of $W^{1, \vartheta}(\Omega)$ and the Eberlein-Smulian theorem imply that $\bar{B}_{\lambda}$ is sequentially weakly compact. The functional $\varphi_{\lambda}(\cdot)$ is sequentially weakly lower semicontinuous (recall that $W^{1, \vartheta}(\Omega) \hookrightarrow L^{p}(\Omega)$ compactly). So, by the Weierstrass-Tonelli theorem, we can find $\widehat{u}_{\lambda} \in W^{1, \vartheta}(\Omega)$ such that

$$
\varphi_{\lambda}\left(\widehat{u}_{\lambda}\right)=\min \left[\varphi_{\lambda}(u): u \in \bar{B}_{\lambda}\right] .
$$

Let $u \in C^{1}(\bar{\Omega}) \subseteq W^{1, \vartheta}(\Omega)$ with $u(z)>0$ for all $z \in \bar{\Omega}$. Then we can find $t \in(0,1)$ small such that $0<t u(z) \leqslant \delta$ for all $z \in \bar{\Omega}$, where $\delta>0$ is as postulated by hypothesis $H_{2}$ (ii). We have

$$
\varphi_{\lambda}(t u) \leqslant \frac{t^{p}}{p} \widehat{\gamma}_{p}(u)+\frac{t^{q}}{q}\|\nabla u\|_{q}^{q}-\widehat{c} t^{\tau}\|u\|_{\tau}^{\tau} \quad \text { (see hypothesis } H_{2}(\mathrm{ii}) \text { ). }
$$

Since $1<\tau<q<p$, choosing $t \in(0,1)$ even smaller if necessary, we have

$$
\begin{aligned}
& \varphi_{\lambda}(t u)<0 \\
& \quad \Rightarrow \quad \varphi_{\lambda}\left(\widehat{u}_{\lambda}\right)<0=\varphi_{\lambda}(0) \quad(\text { see }(25)) \\
& \quad \Rightarrow \quad \widehat{u}_{\lambda} \neq 0 .
\end{aligned}
$$

Also from (24) and (26) it follows that

$$
\left\|\widehat{u}_{\lambda}\right\|<\lambda^{\delta}
$$

Therefore $\widehat{u}_{\lambda} \in B_{\lambda} \backslash\{0\}$. On account of (25) we have

$$
\begin{aligned}
\widehat{u}_{\lambda} & \in K_{\varphi_{\lambda}} \\
& \Rightarrow \widehat{u}_{\lambda} \text { is a nontrivial solution of }\left(P_{\lambda}\right), \lambda \in\left(0, \widehat{\lambda}^{*}\right) .
\end{aligned}
$$

From (27) we see that $\left\|u_{\lambda}\right\| \rightarrow 0^{+}$as $\lambda \rightarrow 0^{+}$. 


\section{References}

[1] P. Baroni, M. Colombo and G. Mingione, Harnack inequalities for double phase functionals, Nonlinear Anal. 121 (2015), 206-222. doi:10.1016/j.na.2014.11.001.

[2] M. Cencelj, V.D. Rădulescu and D.D. Repovš, Double phase problems with variable growth, Nonlinear Anal. 177 (2018), part A, 270-287. doi:10.1016/j.na.2018.03.016.

[3] M. Colombo and G. Mingione, Bounded minimisers of double phase variational integrals, Arch. Ration. Mech. Anal. 218 (2015), 219-273. doi:10.1007/s00205-015-0859-9.

[4] M. Colombo and G. Mingione, Regularity for double phase variational problems, Arch. Ration. Mech. Anal. 215 (2015), 443-496. doi:10.1007/s00205-014-0785-2.

[5] C. De Filippis and G. Mingione, On the regularity of minima for non-autonomous functionals, J. Geom. Anal. doi:10. 1007/s12220-019-00225-z.

[6] L. Gasiński and N.S. Papageorgiou, Constant sign and nodal solutions for superlinear double phase problems, Adv. Calc. Var. doi:10.1515/acv-2019-0040.

[7] B. Ge, D.-J. Lv and J.F. Lu, Multiple solutions for a class of double phase problem without the Ambrosetti-Rabinowitz condition, Nonlinear Anal. 188 (2019), 294-315. doi:10.1016/j.na.2019.06.007.

[8] S. Leonardi and N.S. Papageorgiou, On a class of critical Robin problems, Forum Math. 32 (2020), 95-110. doi:10.1515/ forum-2019-0160.

[9] W. Liu and G. Dai, Existence and multiplicity results for double phase problems, J. Differential Equations 265 (2018), 4311-4334. doi:10.1016/j.jde.2018.06.006.

[10] P. Marcellini, Regularity and existence of solutions of elliptic equations with $p, q$-growth conditions, J. Differential Equations 90 (1991), 1-30. doi:10.1016/0022-0396(91)90158-6.

[11] N.S. Papageorgiou, V.D. Rădulescu and D.D. Repovš, Nonlinear Analysis - Theory and Methods, Springer Nature, Switzerland, 2019.

[12] N.S. Papageorgiou, V.D. Rădulescu and D.D. Repovš, Double-phase problems and a discontinuity property of the spectrum, Proc. Amer. Math. Soc. 147(7) (2019), 2899-2910. doi:10.1090/proc/14466.

[13] N.S. Papageorgiou, V.D. Rădulescu and D.D. Repovš, Positive solutions for nonlinear parametric singular Dirichlet problems, Bull. Math. Sci. 9(3) (2019), 1950011. doi:10.1142/S1664360719500115.

[14] N.S. Papageorgiou, V.D. Rădulescu and D.D. Repovš, Ground state and nodal solutions for a class of double phase problems, Z. Angew. Math. Phys. 71(1) (2020), Paper No. 15. doi:10.1007/s00033-019-1239-3.

[15] N.S. Papageorgiou, C. Vetro and F. Vetro, Multiple solutions with sign information for a $(p, 2)$-equation with combined nonlinearities, Nonlinear Anal. 192 (2020), 111716. doi:10.1016/j.na.2019.111716.

[16] N.S. Papageorgiou, C. Vetro and F. Vetro, Multiple solutions for parametric double phase Dirichlet problems, Commun. Contemp. Math., to appear.

[17] N.S. Papageorgiou and P. Winkert, Applied Nonlinear Functional Analysis, De Gruyter, Berlin, 2018.

[18] M.A. Ragusa and A. Tachikawa, Regularity for minimizers for functionals of double phase with variable exponents, $A d v$. Nonlinear Anal. 9(1) (2020), 710-728. doi:10.1515/anona-2020-0022.

[19] Q. Zhang and V.D. Rădulescu, Double phase anisotropic variational problems and combined effects of reaction and absorption terms, J. Math. Pures Appl. 118 (2018), 159-203. doi:10.1016/j.matpur.2018.06.015.

[20] V.V. Zhikov, Averaging of functionals of the calculus of variations and elasticity theory, Math. USSR Izv. 29 (1987), 33-66. doi:10.1070/IM1987v029n01ABEH000958. 